\title{
Ultrasound Assessment of Ovarian Function Following Radiation Therapy
}

${ }^{1}$ Christopher Prompuntagorn, ${ }^{2} \mathrm{~J}$ Salvador Saldivar, ${ }^{3}$ Sanja Kupesic Plavsic

\begin{abstract}
Ovarian function is important to measure in patients receiving cancer treatments that desire future fertility. Patients with cervical cancer, which affects women of child-bearing age, may be interested in how receiving treatment for the cervical cancer will affect their fertility. Depending on the type and stage of the cancer, women have different options on how to treat the cancer, including radiation, surgery, and chemotherapy. When indicated, radiation therapy carries the risk of ovarian failure in patients wishing to preserve fertility. Ovarian function is measured using hormonal markers and ultrasound markers. Hormonal markers include follicle stimulating hormone (FSH), luteinizing hormone $(\mathrm{LH})$, estradiol, progesterone, inhibin and anti-Müllerian hormone (AMH). It has been shown that in ovarian failure following radiation $\mathrm{FSH}$ and $\mathrm{LH}$ levels are increased; estradiol is increased; inhibin $\mathrm{B}$ is decreased; $\mathrm{AMH}$ is decreased. Ultrasound markers using color Doppler and 3D ultrasound include the resistance index (RI), pulsatility index $(\mathrm{PI})$, vascularization index $(\mathrm{VI})$, flow index $(\mathrm{FI})$, vascularization flow index (VFI) as well as measurements of antral follicle size and count. New developments in ultrasound technology allow us to more reliably than ever assess ovarian function while undergoing radiation treatment.
\end{abstract}

Keywords: Cervical cancer, Radiation, Ovarian function, Hormonal and ultrasound markers, Ovarian volume, Antral follicles, Intraovarian vascularity.

How to cite this article: Prompuntagorn C, Saldivar JS, Kupesic Plavsic S. Ultrasound Assessment of Ovarian Function Following Radiation Therapy. Donald School J Ultrasound Obstet Gynecol 2014;8(3):288-292.

Source of support: Nil

Conflict of interest: None

\section{INTRODUCTION}

Based on the most recent cancer statistics review (Surveillance, Epidemiology and End Results Program—SEER) of the

\footnotetext{
${ }^{1}$ Student Physician, ${ }^{2}$ Associate Professor, ${ }^{3}$ Assistant Academic Dean, Professor

1,2Department of Obstetrics and Gynecology, Paul L Foster School of Medicine, Texas Tech University Health Sciences Center, El Paso, Texas, USA

${ }^{3}$ Faculty Development; Department of Obstetrics and Gynecology, Paul L Foster School of Medicine, Texas Tech University Health Sciences Center, EI Paso, Texas, USA

Corresponding Author: Sanja Kupesic Plavsic, Assistant Academic Dean, Faculty Development; Professor, Department of Obstetrics and Gynecology, Paul L Foster School of Medicine Texas Tech University Health Sciences Center, 4801 Alberta Ave, El Paso, Texas 79905, USA, Phone: 915-215 5065, e-mail: sanja.kupesic@ttuhsc.edu
}

National Cancer Institute, cervical cancer in North America has an incidence of about 5.7 per 100,000, in women mostly of child-bearing age. ${ }^{1,2}$ About 4,000 Americans die of cervical cancer each year. Prevalence of cervical cancer is statistically increased in areas of socioeconomic disparity, and cervical cancer is most prevalent among Hispanic females, a demographic that commonly lacks routine healthcare screenings. ${ }^{3}$

Standard treatments for all stages of cervical cancer may involve extrafascial hysterectomy, radical hysterectomy with pelvic lymphadenectomy or chemosensitizing radiation. ${ }^{4,5}$ Treatment options will depend on the patients age, suitability for major surgery or patient preference. ${ }^{6}$ In a subset of patients wishing to preserve fertility (i.e. future surrogacy), those undergoing radiation therapy may choose ovarian transposition and oophoropexy in order to reduce the risk of ovarian failure. Some studies have shown laparoscopic transposition to preserve normal ovarian function in about $88 \%$ of women undergoing pelvic irradiation. ${ }^{7}$ However, some women opt to begin radiotherapy without undergoing a surgical procedure.

Ovarian failure is one of the risks of receiving radiation therapy to the pelvis due to the destruction of the oocyte pool, also known as ovarian reserve, by the radiation. ${ }^{8,9}$ Radiation exposure greater than 24 Gy can cause premature ovarian failure ${ }^{10}$ and the amount of radiation to cause oocyte depletion is inversely related to the patient's age at the time of therapy. ${ }^{11}$ Some studies have demonstrated that as little as $9 \mathrm{~Gy}$ can cause premature ovarian failure. ${ }^{9}$ However, the cervix is exposed to 25 fractions of around 2 Gy per day for a total of approximately $50 \mathrm{~Gy}$ over the treatment. ${ }^{12}$ Depending on the inverse square of the distance from the radiation, the exposure to radiation may make future pregnancy less probable, but not impossible. ${ }^{9,13}$

\section{HORMONAL MARKERS OF OVARIAN FUNCTION}

In women with normal ovarian function, the menstrual cycle occurs regularly on a monthly basis. The hypothalamicpituitary-ovarian axis is stable, with the gonadotropin stimulation of ovarian hormones occurring in a feedback loop, with the gonadotropins, follicle stimulating hormone (FSH) and luteinizing hormone (LH), initiating a reactive change in levels ovarian hormones, estradiol, progesterone, and inhibin. ${ }^{14}$ The ovarian changes during the menstrual 
cycle depend on FSH and LH secreted by the anterior pituitary gland. The levels of sex hormones cycle up and down between the follicular phase and the luteal phase, with ovulation occurring in between. In the follicular phase, FSH and LH levels increase slightly which leads to proliferation of granulosa cells and theca cells, which will lead to increased production of estradiol (levels of which are higher than that of progesterone) as well as development of the follicles. Immediately prior to ovulation, the levels of LH increase markedly, and this spike leads to release of the oocyte; the LH spike will also change the granulosa and theca cells to lutein cells primarily serving to produce progesterone, making progesterone levels rise higher than estradiol for the duration of the luteal phase. ${ }^{15}$

The ovaries also produce anti-Müllerian hormone $(\mathrm{AMH})$ whose levels are independent of the gonadotropin levels; ${ }^{14,16}$ AMH levels vary minimally throughout the menstrual cycle, slightly increasing during the luteal phase. ${ }^{17}$ $\mathrm{AMH}$ is secreted by granulosa cells of preantral and antral follicles, mainly from follicles measuring less than $6 \mathrm{~mm}$ in diameter. ${ }^{18}$ Other studies have shown that follicles 5 to $8 \mathrm{~mm}$ in diameter produce an estimated AMH levels decrease as the development of follicles progresses; therefore, $\mathrm{AMH}$ levels can predict a woman's ovarian reserve. Some studies have shown that AMH is the most reliable hormonal marker in predicting ovarian reserve as it relates to ovarian response in the context of in vitro fertilization (IVF). ${ }^{19}$

Menopause is defined as the permanent cessation of menstrual periods, defined as 12 months of amenorrhea without a separate organic cause. Of the hormones involved in the menstrual cycle, follicle stimulating hormone levels have been reliably used to measure the STRAW (stages of reproductive aging workshop) stage of ovarian function as women approach menopause. ${ }^{20,21}$ During the perimenopausal period, FSH levels increase as declining levels of inhibin are no longer able to maintain the negative feedback. $^{22}$ Follicular phase FSH levels will increase from around $20 \mathrm{IU} / \mathrm{L}$ in child-bearing years to around the 70 to $100 \mathrm{IU} / \mathrm{L}$ range after menopause. ${ }^{23}$

Premature ovarian failure, like that which may be caused by radiation therapy, will demonstrate similar physiologic changes as in menopause at an earlier age. One study demonstrated that childhood cancer survivors following radiation therapy had a significantly increased early follicular phase FSH levels compared to controls of similar age. ${ }^{24}$

Ovarian function can be quantified by measuring gonadotropins FSH and $\mathrm{LH}$ as well as estradiol, antiMüllerian hormone, ${ }^{16}$ and inhibin B. In cases of premature ovarian failure following radiation therapy, FSH and $\mathrm{LH}$ levels are increased; estradiol is increased; inhibin $\mathrm{B}$ is decreased; ${ }^{25}$ AMH is decreased. ${ }^{26}$

\section{ULTRASOUND MARKERS OF OVARIAN FUNCTION}

Pelvic sonography can measure ovarian function by visualizing the follicles to determine the follicle count. The size of the ovary can be quantified as well. In post-radiotherapy patients, both ovarian size and follicle count are decreased.

Transvaginal ultrasound is considered the most reliable method for monitoring the follicular growth and detection of ovulation. ${ }^{27}$ Addition of color Doppler enables visualization of the extrinsic and intrinsic ovarian vascular changes throughout the menstrual cycle (Figs 1 and 2). Follicular blood flow can also help, to determine the timing of ovulation in the menstrual cycle. ${ }^{27}$ The basic structural information provided by conventional scans in the longitudinal and transverse plan can now be augmented by the use of three-dimensional (3D) ultrasound systems that provide additional views of the coronal plane, which is parallel to the transducer face. The computer-generated scan is displayed in three perpendicular planes which allow precise estimation of the ovarian volume. ${ }^{27}$ Transvaginal two-dimensional (2D) and $3 \mathrm{D}$ sonography with color flow mapping can assess the ovarian function by measuring the ovarian size and volume, visualizing the number of antral follicles to determine the follicle count and evaluating the quality intraovarian perfusion (Fig. 3). In postradiotherapy patients, both the ovarian size and follicle count are decreased, and intraovarian blood flow signals are usually absent (Fig. 4).

During reproductive years, the ovary volume determined by ultrasound is relatively stable. The mean ovarian volume is above $6 \mathrm{~cm}^{3}$ in reproductive-aged women; in the perimenopausal period, the ovarian volume is about $4.9 \mathrm{~cm}^{3}$; while after menopause, the volume is significantly decreased to around $2 \mathrm{~cm}^{3}{ }^{28}$ In a study of cancer survivors that had undergone

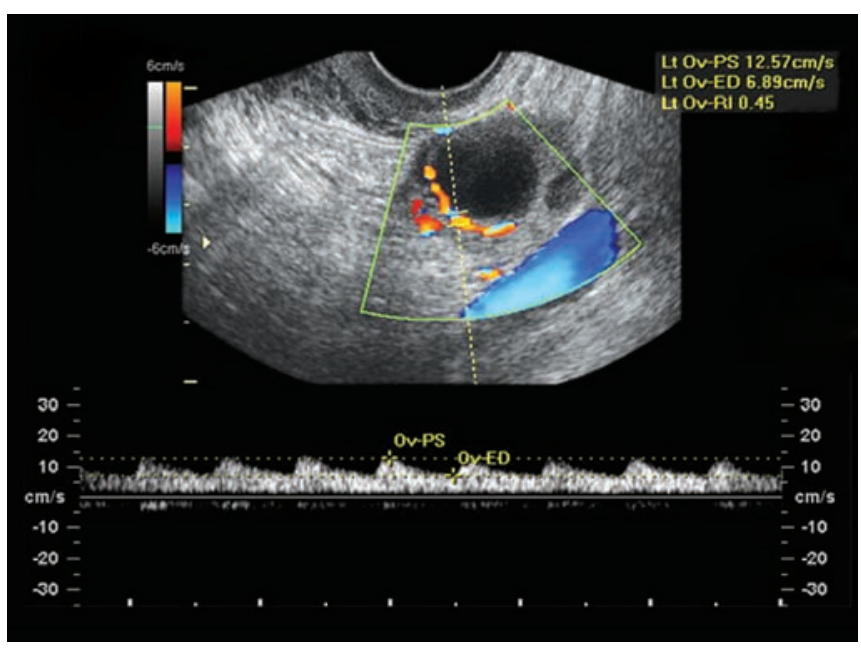

Fig. 1: Transvaginal color Doppler ultrasound of the preovulatory follicle. Perifollicular vessels are clearly displayed. Pulsed Doppler waveform analysis shows a resistance index of 0.45 . Iliac vessels are seen below the ovary 
radiation, mean ovarian volume was measured at $3 \mathrm{~cm}^{3}$ in the cancer survivor group vs $5 \mathrm{~cm}^{3}$ in the control group. ${ }^{24}$

The use of color Doppler ultrasound facilitates detection of the intraovarian vascularity and enables objective analysis of the vascular impedance to assess ovarian function and events in the menstrual cycle. ${ }^{29}$ The ovary has a dual blood supply: the ovarian artery and the utero-ovarian branch of the uterine artery which eventually anastomose with one another. These vessels pass through the medullary portion of the ovarian stroma toward the cortex where they create a vascular bed surrounding the follicles. It is possible to visualize the anatomic changes in these vessels via color Doppler, during both the follicular and luteal phases of the menstrual cycle. ${ }^{27}$ Color Doppler is a useful modality to visualize the intricate ovarian vascular system and assess the vascular resistance of the perifollicular and corpus luteum

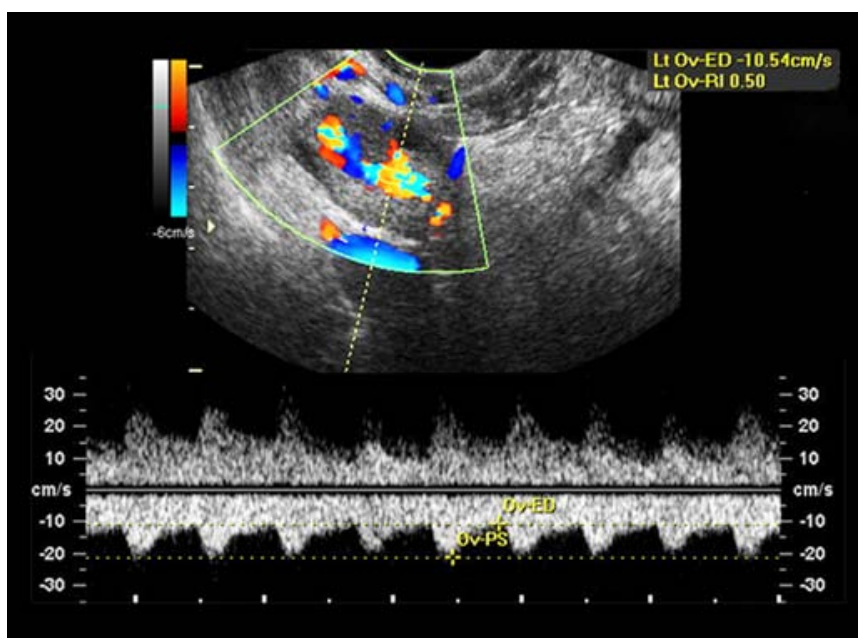

Fig. 2: Transvaginal color Doppler ultrasound of the ovary in midluteal phase. Increased vascularity of the corpus luteum is easily seen by color flow mapping. A high blood flow velocity and low resistance index $(R I=0.50)$ represent typical flow pattern of the corpus luteum

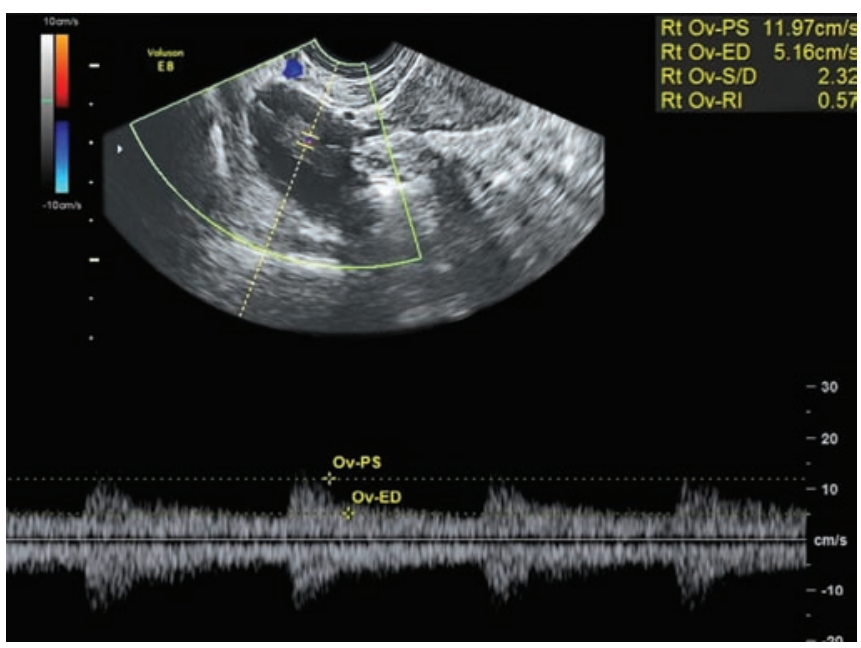

Fig. 3: Baseline transvaginal color Doppler sonogram of the normal size ovary in reproductive age patient. Multiple antral follicles, normal ovarian volume and moderate vascular resistance (RI 0.57) indicate normal ovarian function vessels. In ovulatory women during the periovulatory period, there is a progressive reduction in vascular resistance of the perifollicular vessels (see Fig. 1). In addition, the blood flow velocity increases significantly 24 hours before follicular rupture and remains elevated during the entire luteal phase (see Fig. 2). ${ }^{30}$ There is a relationship between the age and decreased Doppler indices for ovarian stromal vascularity and blood flow, ${ }^{31}$ which in turn correlates to a decrease in the number of antral follicles. ${ }^{32}$

Three-dimensional power Doppler ultrasound can objectively measure intraovarian vascularity by expressing the vascularization index (VI), flow index (FI) and vascularization flow index (VFI). Vascularization index represents the percentage of blood vessels in ovaries using the percentage of color voxels (three-dimensional equivalent of pixels) among total voxels in the volume of interest to represent the areas where blood vessels are present. The value of color of those voxels corresponds to the intensity of flow, and the mean value of the colors is used as a flow index. Furthermore, multiplying the VI and FI produces a vascularization flow index. ${ }^{33}$ One study showed the annual decrease in total ovarian VI is around $0.18 \%$ using a linear regression model. ${ }^{31}$ Another study showed that undetectable blood flow to ovarian stroma was significantly related to a low ovarian reserve; 9/15 women with low ovarian reserve compared to $1 / 17$ with good ovarian reserve showed undetectable flow. ${ }^{34}$

New developments in three-dimensional ultrasound are able to reliably quantify the number of follicles as well as the volume of those follicles. ${ }^{35,36}$ While a decrease in ovarian volume is related to aging and decreasing ovarian function, the antral follicle count is the quantification study of choice to measure ovarian function. ${ }^{37}$ The size and number of antral follicles declines with age, consistent with the decreasing the

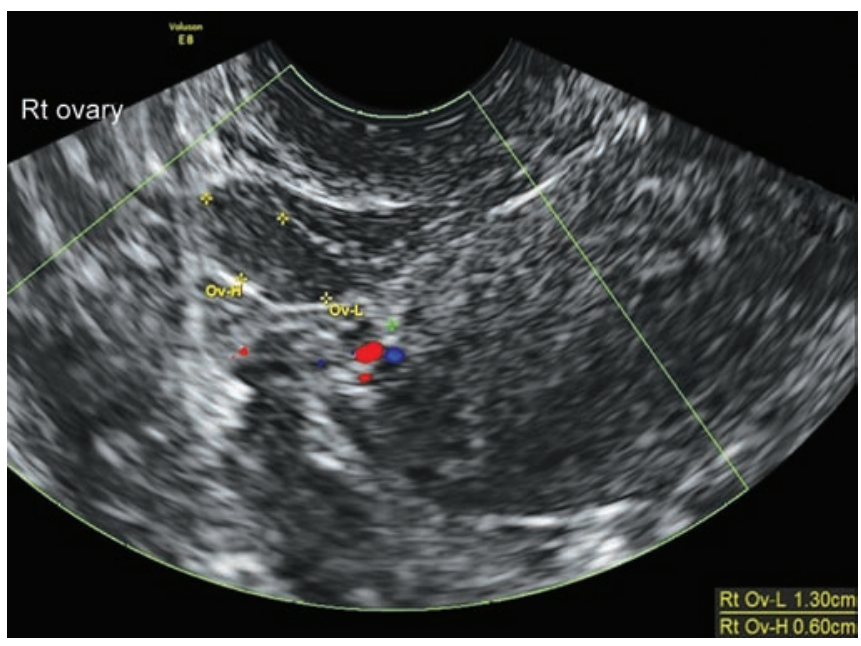

Fig. 4: Transvaginal color Doppler sonogram of a patient with premature ovarian failure following radiotherapy for cervical cancer. Note absence of antral follicles, decreased ovarian volume and absence of intraovarian flow 
ovarian volume. ${ }^{38}$ Smaller antral follicles may be indicative of a decreased amount of the remaining primordial follicle pool in women approaching menopause. ${ }^{39}$ Furthermore, menstrual cycle-dependent changes are more likely to affect the counts of the larger ( $>6 \mathrm{~mm}$ ) follicles yet minimally affecting the counts of the smaller follicles. ${ }^{17}$ As mentioned earlier, follicles less than $6 \mathrm{~mm}$ in diameter are associated with $\mathrm{AMH}$ secretion and the amount of those follicles is predictive of the oocyte reserve. ${ }^{40}$

\section{CONCLUSION}

By applying the knowledge of ovarian function as it relates to premature ovarian failure, we can potentially use the ultrasound markers to assess immediate effects on the ovary following pelvic radiation therapy for cervical cancer. New measures of ultrasound imaging can provide reliable data on how the ovary is immediately affected by the radiation which may allow us to prognosticate future fertility of cervical cancer patients undergoing radiotherapy.

\section{REFERENCES}

1. Jemal A, Bray F, Center MM, Ferlay J, Ward E, Forman D. Global cancer statistics. CA. A Cancer Journal for Clinicians 2011;61(2):69-90.

2. Singh GK, Miller BA, Hankey BF, Edwards BK. Persistent area socioeconomic disparities in US incidence of cervical cancer, mortality, stage, and survival, 1975-2000. Cancer 2004; 101(5):1051-1057.

3. Bazargan M, Bazargan SH, Farooq M, Baker RS. Correlates of cervical cancer screening among underserved Hispanic and African-American women. Preventive Medicine 2004;39(3): 465-473.

4. Landoni F, Maneo A, Colombo A, Placa F, Milani R, Perego P, et al. Randomised study of radical surgery versus radiotherapy for stage Ib-IIa cervical cancer. The Lancet 1997;350(9077):535-540.

5. National Cancer Institute: $\mathrm{PDQ}^{\circledR}$ Cervical Cancer Treatment. Bethesda, MD: National Cancer Institute; 2014 [(updated 3/14/ 2014; cited 2014); Health Professional Version]. Available at: cancer.gov/cancertopics/pdq/treatment/cervical/health Professional.

6. Burghardt E, Baltzer J, Tulusan AH, Haas J. Results of surgical treatment of 1028 cervical cancers studied with volumetry. Cancer 1992 Aug 1;70(3):648-655.

7. Bisharah M, Tulandi T. Laparoscopic preservation of ovarian function: An underused procedure. American Journal of Obstetrics and Gynecology 2003;188(2):367-370.

8. van Kasteren Y, Schoemaker J. Premature ovarian failure: a systematic review on therapeutic interventions to restore ovarian function and achieve pregnancy. Human Reproduction Update 1999 September 1;5(5):483-492.

9. Goswami D, Conway GS. Premature ovarian failure. Human Reproduction Update 2005 July 1;11(4):391-410.

10. Stroud JS, Mutch D, Rader J, Powell M, Thaker PH, Grigsby PW. Effects of cancer treatment on ovarian function. Fertility and Sterility 2009;92(2):417-427.

11. Wallace WHB, Thomson AB, Saran F, Kelsey TW. Predicting age of ovarian failure after radiation to a field that includes the ovaries. International Journal of Radiation Oncology Biology Physics 2005;62(3):738-744.

12. Nag S, Orton C, Young D, Erickson B. The American Brachytherapy Society Survey of Brachytherapy Practice for Carcinoma of the Cervix in the United States. Gynecologic Oncology 1999;73(1):111-118.

13. Francis $\mathrm{O}$, Stevens RD. Pregnancy after Primary Irradiation for Carcinoma of Cervix. Br Med J 1965 Aug 7;2(5457):342-343.

14. Burger HG, Hale GE, Robertson DM, Dennerstein L. A review of hormonal changes during the menopausal transition: focus on findings from the Melbourne Women's Midlife Health Project. Human Reproduction Update 2007 November 1, 2007; 13(6):559-565.

15. Hall JE, Guyton AC. Female Physiology before Pregnancy and Female Hormones. Chapter 81: Guyton and Hall Textbook of Medical Physiology. 12th ed. Philadelphia, PA, USA; Saunders Elsevier 2011;987-1002.

16. Visser JA, de Jong FH, Laven JS, Themmen AP. Anti-Müllerian hormone: a new marker for ovarian function. Reproduction 2006 Jan;131(1):1-9.

17. Deb S, Campbell BK, Clewes JS, Pincott-Allen C, RaineFenning NJ. Intracycle variation in number of antral follicles stratified by size and in endocrine markers of ovarian reserve in women with normal ovulatory menstrual cycles. Ultrasound in Obstetrics and Gynecology 2013;41(2):216-222.

18. Deb S, Kannamannadiar J, Campbell BK, Clewes JS, RaineFenning NJ. The interovarian variation in three-dimensional ultrasound markers of ovarian reserve in women undergoing baseline investigation for subfertility. Fertility and Sterility 2011;95(2):667-672.

19. van Rooij IAJ, Broekmans FJM, te Velde ER, Fauser BCJM, Bancsi LFJMM, Jong FHd, et al. Serum anti-Müllerian hormone levels: a novel measure of ovarian reserve. Human Reproduction 2002 December 1;17(12):3065-3071.

20. Soules MR, Sherman S, Parrott E, Rebar R, Santoro N, Utian W, et al. Executive summary: stages of reproductive aging workshop (STRAW). Fertility and Sterility 2001;76(5):874-878.

21. Su HI, Freeman EW. Hormone changes associated with the menopausal transition. Minerva Ginecol 2009 Dec;61(6):483-489.

22. Hall JE. Neuroendocrine physiology of the early and late menopause. Endocrinol Metab Clin North Am 2004 Dec;33(4): 637-659.

23. Randolph JF, Zheng H, Sowers MR, Crandall C, Crawford S, Gold EB, et al. Change in follicle-stimulating hormone and estradiol across the menopausal transition: effect of age at the final menstrual period. The Journal of Clinical Endocrinology and Metabolism 2011;96(3):746-754.

24. Bath LE, Wallace WHB, Shaw MP, Fitzpatrick C, Anderson RA. Depletion of ovarian reserve in young women after treatment for cancer in childhood: detection by anti-Müllerian hormone, inhibin B and ovarian ultrasound. Human Reproduction 2003 Nov 1;18(11):2368-2374.

25. Larsen EC, Müller J, Schmiegelow K, Rechnitzer C, Andersen AN. Reduced ovarian function in long-term survivors of radiation- and chemotherapy-treated childhood cancer. The Journal of Clinical Endocrinology and Metabolism 2003;88(11):5307-5314.

26. van Beek RD, van den Heuvel-Eibrink MM, Laven JSE, de Jong FH, Themmen APN, Hakvoort-Cammel FG, et al. Anti-Müllerian hormone is a sensitive serum marker for gonadal function in women treated for Hodgkin's lymphoma 
during childhood. The Journal of Clinical Endocrinology and Metabolism 2007;92(10):3869-3874.

27. Kupesic S, Kurjak A. Color Doppler and three-dimensional ultrasound imaging in infertility. Color Doppler, 3D and 4D ultrasound in gynecology, infertility and obstetrics. 2nd ed. New Delhi: Jaypee Brothers Medical Publishers 2011. p. 115-130.

28. Pavlik EJ, DePriest PD, Gallion HH, Ueland FR, Reedy MB, Kryscio RJ, et al. Ovarian volume related to age. Gynecologic Oncology 2000;77(3):410-412.

29. Jokubkiene L, Sladkevicius P, Rovas L, Valentin L. Assessment of changes in volume and vascularity of the ovaries during the normal menstrual cycle using three-dimensional power Doppler ultrasound. Human Reproduction 2006 October 1;21(10):26612668.

30. Kurjak A, Kupesic-Urek S, Schulman H, Zalud I. Transvaginal color Doppler in the assessment of ovarian and uterine blood flow in infertile women. Fertil Steril 1991;56(5):870-873.

31. Ng EHY, Chan CCW, Yeung WSB, Ho PC. Effect of age on ovarian stromal flow measured by three-dimensional ultrasound with power Doppler in Chinese women with proven fertility. Human Reproduction 2004 Sep 1;19(9):2132-2137.

32. Kupesic S, Kurjak A. Predictors of IVF outcome by threedimensional ultrasound. Human Reproduction 2002 Apr 1;17(4): 950-955.

33. Alcázar JL. Three-dimensional power Doppler derived vascular indices: what are we measuring and how are we doing it? Ultrasound in Obstetrics and Gynecology 2008;32(4):485-487.

34. Younis JS, Haddad S, Matilsky M, Radin O, Ben-Ami M. Undetectable basal ovarian stromal blood flow in infertile women is related to low ovarian reserve. Gynecological Endocrinology 2007;23(5):284-289.

35. Jokubkiene L, Sladkevicius P, Valentin L. Number of antral follicles, ovarian volume, and vascular indices in asymptomatic women 20 to 39 years old as assessed by three-dimensional sonography: a prospective cross-sectional study. Journal of Ultrasound in Medicine 2012 Oct 1;31(10):1635-1649.

36. Jayaprakasan K, Campbell BK, Clewes JS, Johnson IR, RaineFenning NJ. Three-dimensional ultrasound improves the interobserver reliability of antral follicle counts and facilitates increased clinical work flow. Ultrasound in Obstetrics and Gynecology 2008;31(4):439-444.

37. Hendriks DJ, Kwee J, Mol BWJ, te Velde ER, Broekmans FJM. Ultrasonography as a tool for the prediction of outcome in IVF patients: a comparative meta-analysis of ovarian volume and antral follicle count. Fertility and Sterility 2007;87(4): 764-775.

38. Kurjak A, Kupesic S, Simunic V. Ultrasonic assessment of the peri- and postmenopausal ovary. Maturitas 2002;41(4):245-254.

39. Kupesic S, Kurjak A, Bjelos D, Vujisic S. Three-dimensional ultrasonographic ovarian measurements and in vitro fertilization outcome are related to age. Fertility and Sterility 2003;79(1): 190-197.

40. Jayaprakasan K, Deb S, Batcha M, Hopkisson J, Johnson I, Campbell B, et al. The cohort of antral follicles measuring 2 to $6 \mathrm{~mm}$ reflects the quantitative status of ovarian reserve as assessed by serum levels of anti-Müllerian hormone and response to controlled ovarian stimulation. Fertility and Sterility 2010; 94(5):1775-1781. 